\title{
Research on the Value Application of Fishbone Diagram Analysis in the Management and Control of Wound Infection in Operating Room
}

\author{
Changxia Cheng, Yingmei Qi *, Huan Zhang
}

Department of Surgery, China - Japan Friendship Hospital, Jilin University, Jilin, 130000, China

*corresponding author’s email: 1021161418@qq.com

Keywords: Fish Bone Diagram Analysis; Wound Infection; Control and Management; Value

\begin{abstract}
Objective: To discuss the value of fish bone diagram analysis application in wound infection control and management of operating room. Method: 1200 cases of surgical treatment patients treated in our hospital from January 2010 to December 2014 were chosen, and 580 cases were chosen before Fishbone Analysis Manager, 620 cases were chosen after manager. Factors affecting wound infection after surgery of the two groups were analyzed, and the corresponding control method is summarized. The impact of the operating room wound infection "infection factors Fish bone" and "Control Manager Fish bone" is drawn out according to the reasons. The fishbone diagram is implemented. And 15 nurses of the operating room were given control and management training, Theory examination results and wound infection of patients before and after the training were observed. Results: After the control and management of fishbone diagram analysis application in wound infection of operating room, Theory test results of nurses were significantly improved compared to the previous, wound infection was significantly lower in patients $(\mathrm{P}<0.05)$. Conclusion: Fishbone diagram analysis application in wound infection control and management of operating room can enable nurses to better grasp the theory of knowledge, reduce the incidence of wound infection and is worthy of popularization.
\end{abstract}

\section{Introduction}

Infection refers to the local or systemic inflammatory response of the human body under the invasion of various pathogens such as bacteria, fungi, viruses, etc. [1]. For patients undergoing surgery, postoperative incision infections cause great problems for patients and medical personnel, which seriously affects the rehabilitation of patients [2]. Not only bring great pain to patients, delay the recovery of patients, but also bring a heavy financial burden to family members [3]. Microorganisms are ubiquitous in the human body and its surroundings. When doctors, patient surgical areas, surgical related equipment, and dressings are not thoroughly disinfected, it is easy to cause infection in the surgical incision. Therefore, only by constantly looking for the cause of the infection, the patient's susceptibility factors, and taking targeted control and management measures, can minimize the incidence of infection and improve the patient's treatment effect [4]. With the rapid development of the economy and society and the advancement of modern medicine, there are more and more precision instruments in the operation. As the amount of surgery increases, the patient's expectation for the treatment effect increases, and the infection of the operating room incision must be systematic. Standardized control and management make the operation safe and smooth [5]. In this study, 1200 patients with various diseases treated in our hospital were selected as the research object. The influencing factors of postoperative incision infection were analyzed. The influencing factors of incision infection in the operating room were analyzed, and the corresponding control methods were summarized. The value of fishbone diagram analysis is in the management of incision infection management in the operating room. The results of the analysis are now reported as follows: 


\section{Materials and Methods}

\subsection{General Information.}

A total of 1200 patients with various diseases who underwent surgery in our hospital from January 2010 to December 2014 were selected as subjects. Among them, 580 patients were managed before fish bone analysis and 620 patients were managed. The 15 nurses in the operating room of our hospital were selected for training, all female, aged 35-51 years, mean (42.8 \pm 2.1$)$ years old. Education: 9 undergraduates, 6 junior colleges. Title: 12 supervisors and 3 deputy directors.

\subsection{Management Methods.}

Assessment of the status quo: The investigation of the causes of infection in the operating room and the mastery of the theoretical knowledge of the nursing staff revealed that the following problems existed in the control of the infection of the incision: the nurse's control management procedure and target for the infection of the wound after surgery are not clear Nurses can not well grasp the causes of postoperative incision infection; nurses have less awareness of protection; the workload is larger; the result is an increase in the work and psychological stress of medical staff.

Influential factors of infection in the operating room: 1 Personnel: The oldest patient is 95 years old, the youngest is only 5 years old, the average age of the patient is 59 years old; due to the older age, the body's resistance Reduced, it is easy to get infected. At the same time, due to the irregular operation of the medical staff during the operation, the low sense of responsibility, and the greater mobility of some surgical personnel, the chance of postoperative wound infection is increased. 2 surgical materials: due to the rapid update of surgical equipment, high cost, some disposable consumables are reused. At the same time, the material supplier did not follow the relevant regulations of the country to conduct standardized operations and contaminate raw materials. The application of human implants is arbitrarily and improperly received and stored. The relevant consumables used in the operation were not thoroughly sterilized before entering the operating room. 3 Operating room environment: Due to the huge cost of cleaning the operating room, the filter was not replaced on time; the vents and pipes were not thoroughly cleaned, and the areas were not standardized. 4 surgical instruments, dressings, instruments: the equipment in the operating room are more expensive, meticulous, and the number of spares is limited. The hospital often uses an autoclave for rapid sterilization when sterilizing surgical instruments. Rapid cleaning and disinfection of the device often results in inadequate disinfection. Due to the large number of instruments in the operating room, cross-contamination can occur. 5 Management system related to the operating room: The management system for the entry and exit of the operating room, the surgical staff, etc. is not perfect, some of the loopholes exist, the material collection and storage system is not perfect, and the protection system for infectious diseases is not thorough. 6 The nurses in the operating room have a mastery of knowledge: the nurses have a weak grasp of the knowledge about the wound infection, and the assessment results are low.

Relevant measures taken for influencing factors: 1 Personnel: Patients should fully improve the preoperative examination before surgery to exclude patients with local infection. The staff in the operating room must dress according to the regulations. The patient can complete the preoperative preparation in the ward and then arrive at the operating room under the transfer of the special surgical vehicle. Masks, hats, and underwear in the operating room must be placed in a predetermined position. When selecting a garment, materials that are not easily detached from the fibers and that are less likely to generate static electricity should be selected. Operators in the operating room should be guaranteed to be within the specified range. The medical staff strictly observes the aseptic operation during the operation. 2 Surgical materials: The materials in the operating room should be managed by a person and set up an independent warehouse; when the raw materials are purchased, the supplier is strictly required to eliminate the purchase of unqualified products. All materials pass through the dedicated passage of the operating room and are disinfected and wiped into the operating room warehouse. For the one-time materials, the corresponding registration book should be established and used by one person to avoid the occurrence of repeated 
use. 3 operating room environment: the operation of the same operating room should be arranged in a reasonable order, the maximum self-cleaning time of the operating room is extended. Regularly clean, disinfect and purify the equipment piping, and regularly replace the filter as required. After the completion of the cleaning work every day, the operating room purification system should continue to run for 15 20 minutes, then the laminar air conditioner should be turned off to ensure the indoor air is clean. All areas in the operating room should be clearly marked to increase the buffer area to ensure complete and orderly facilities. 4 surgical instruments, dressings, instruments: all kinds of instruments, equipment should be installed before being sent to the operating room, and wiped clean. Before and after surgery, the shadowless lamp, microscope, instrument vehicle, operating bed, etc. should be wiped once with disinfectant. The above equipment should be thoroughly wiped and maintained once a week. The autoclave should be standardized according to the regulations, and the relevant chemical and biological monitoring should be carried out. The registration work should be carried out, and the sterilization should be carried out according to the sterilization method of each equipment. Medical staff should perform surgery in strict accordance with the requirements of aseptic operation to prevent cross-infection. 5 operating room related management system: constantly improve the relevant management system, according to the actual situation of the operation to develop surgery and nursing staff, handing over patients, items in and out, infectious disease isolation and other systems, select one person in the operating room nurses for operating room infection control Management work. 6 The nurses in the operating room have knowledge of the knowledge: Explain and analyze the fishbone diagram of the factors related to the infection of the operating room in the 15 nurses, so that the nursing staff can fully understand the meaning of the relevant indicators in the fishbone diagram, and comprehensively grasp the incision. In turn, the nursing staff is analyzed for the attention during the operation, so that they can master and control the various tasks in the fishbone diagram.

\subsection{Observation Indicators.}

Observe and record the theoretical assessment scores of the nurses before and after the training and the number of incision infections of the patients.

\subsection{Statistical Analysis.}

The data were statistically analyzed using SPSS 17.0. The measurement data were expressed as mean \pm standard deviation $( \pm s)$. The t-test was used, and the count data was used. The test was performed, and the difference was statistically significant at $\mathrm{P}<0.05$.

\section{Results}

Appraisal results of nurses before and after applying fishbone diagram control management. After the fishbone analysis method was used to control the infection of the operating room incision, the nurses' theoretical examination scores were significantly improved, and the difference was statistically significant $(\mathrm{P}<0.05)$. The specific results are shown in Table 1:

Table 1 Comparison of results before and after applying fishbone diagram (minutes, $\pm \mathrm{s}$ )

\begin{tabular}{llc}
\hline Group & Number of people & $\begin{array}{c}\text { Theoretical } \\
\text { achievement }\end{array}$ \\
\hline Before intervention & 15 & $82.15 \pm 5.25$ \\
After intervention & 15 & $96.31 \pm 3.13$ \\
$t$ & $/$ & 8.364 \\
$P$ & $/$ & 0.001 \\
\hline
\end{tabular}

Application of fishbone diagram to control the infection of patients before and after management. After the fishbone analysis was used to control the infection of the incision in the operating room, the infection rate of the incision was significantly reduced, and the difference was statistically significant $(\mathrm{P}<0.05)$. The specific results are shown in Table 2: 
Table 2 Comparison of adverse reaction results before and after application of fishbone diagram [n(\%)]

\begin{tabular}{lll}
\hline Group & Number of people & Infection \\
\hline Before intervention & 580 & $93(16.0)$ \\
After intervention & 620 & $36(5.8)$ \\
$\chi^{2}$ & $/$ & 6.342 \\
$P$ & $/$ & 0.0021 \\
\hline
\end{tabular}

\section{Discussion}

Fishbone diagram analysis can help nurses grasp the cause of infection in the operating room. The fishbone diagram analysis method is also called causal analysis method [6]. This method can be used to find the root cause of things, because the shape is very similar to the bones of fish. So it is called a fishbone map. The fishbone diagram can be used to intuitively propose problems and various reasons. Through everyone's brainstorming and giving full play to the wisdom of group cooperation, we can find out all the causes of the problem or a related component from various angles [7]. Traditional nurse training often gives a training model, but because nurses always memorize the relevant knowledge, they can not have a systematic and comprehensive understanding and understanding of theoretical knowledge, even though it was remembered at the time, it is easy forget. Related studies have shown that the risk of postoperative incision infection in emergency surgery patients is higher than that in elective surgery patients [8]. From Figure 1, we can find that there are many risk factors for wound infection in surgical patients. During the operation, although nurses can cooperate well with surgery and pay attention to infection management and prevention, nurses will inevitably have some omissions in some aspects. In explaining the cause of infection in the operating room, the explanation with the fishbone diagram shows that the advantages of the veins are clear and the ideas are clear, which can help the nurses to grasp the relevant risk factors comprehensively.

Operating room incision infection control management. The application of fishbone diagram can effectively help nurses control related infection factors. The operating room is one of the important departments of the hospital. It is the largest surgical treatment site in the hospital and plays a major role in controlling hospital infection [9]. According to Figure 1, we can see that there are six reasons for infection in the operating room, namely, personnel, surgical materials, operating room environment, surgical instruments, dressings, instruments, operating room management systems, and operating room nurses. In each type of reason, there are other predisposing factors that cause this cause. We draw the relevant reasons into the fishbone diagram of the infectious factor, which can make the nurse see at a glance, reduce the difficulty of the nurse's memory, and deepen the pair. Infectious factors understand and master the fishbone diagram [10]. Therefore, the use of infectious factors fishbone diagrams in the operation of patients can enable nurses to follow the rules, not to miss some infectious factors and improve the quality of care [11]. The results of this study showed that after the fishbone analysis method was used to control the infection of the incision in the operating room, the infection rate of the incision was significantly reduced, and the difference was statistically significant $(\mathrm{P}<0.05)$.

Fishbone diagram analysis can significantly improve the learning efficiency of nurses. Because there are many operations performed every year in our hospital, the infection factors of the surgery are analyzed every year and the nursing staff are assessed and trained. However, due to more infection factors and various training measures, it is difficult for nurses to master relevant knowledge comprehensively, so the theoretical evaluation results are not satisfactory [12-13]. Since the development of fishbone diagrams and related training for nurses, the efficiency of nurses has been greatly improved, and the assessment results of nurses have been improved. The results of this study show that after the fishbone analysis method is used to control and manage the wound infection in the operating room, The nurses' theoretical assessment scores were significantly improved, and the differences were statistically significant $(\mathrm{P}<0.05)$. 
Fishbone diagram analysis can effectively strengthen the communication of medical staff and standardize medical behavior. During the operation of the patient, the nurse in the operating room is the leader in infection control management and has the responsibility to supervise the operation of the surgeon and the anesthesiologist. Some doctors operate irregularly during the operation, the used surgical instruments are not returned in time, causing the patient to have a stab wound, and the anesthesiologist does not return the needle to the sharp box in time after the puncture, which may lead to the occurrence of postoperative wound infection. The rate increases. After using the fishbone diagram analysis method, the medical staff's awareness of aseptic operation has been significantly improved. The nurses have clearly defined their responsibilities and actively communicated with the surgeons and anesthesiologists, fully demonstrating the spirit of collaboration between the teams and increasing. The cohesiveness between the teams reduces the incidence of infection in the operating room.

To sum up: the application of fishbone diagram analysis in the management of wound infection control in the operating room can enable the nursing staff to better grasp the relevant theoretical knowledge and reduce the incidence of wound infection, which is worthy of clinical promotion.

\section{References}

[1] Zhou Ping. Application of fish bone analysis method in emergency triage care [J]. Chinese Journal of Coal Industry Medicine, 2015, (04): 669-671.

[2] Guo Guang, Qiao Xiaochun analysis of risk factors related to surgical incision infection [J]. Chinese Journal of Hospital Infectious Diseases, 2013, (16): 3891-3893.

[3] ZHANG Jing, SHI Jing-fen, YAN Ya-ping. Factors and countermeasures influencing clinical nursing teaching in respiratory department using fishbone diagram [J]. Nursing Practice and Research, 2013, (23): 65-67.

[4] Chen Nali, Zhang Hua, Hu Chunrong. Analysis of the causes of occlusion of elderly patients with central venous catheter by peripheral vein using fishbone diagram and nursing countermeasures [J]. Journal of Bengbu Medical College, 2013, (09): 1227-1229.

[5] Liu Yongxiang. Strengthening the management of operating room to control hospital infections [J]. Chinese Practical Medicine, 2008, (09): 146-147.

[6] Zhao Lihua, Liu Geng, Wang Jingbo. Effects and control of operating room management on surgical wound infection [J]. Chinese Journal of Nosocomiology, 2015, (01): 213-215.

[7] Jin Peng, Li Binxin, Sun Lili. Discussion on the role of operating room nursing management in controlling nosocomial infections [J]. Chinese Journal of Pharmaceutical Economics, 2014, (03): 332-333.

[8] Song Shuhua. Application of operation room detail nursing management in controlling hospital infection [J]. Nursing Practice and Research, 2014, (02): 89-90.

[9] He Qinxiang. Discussion on the application of nursing management in controlling nosocomial infection [J].Jilin Medical Journal, 2010,(35):6571.

[10] WANG Yan,ZHANG Aihua.Influence and control of operating room management on surgical wound infection[J].Practical Preventive Medicine, 2013,(09): 1127-1128.

[11] Huang Sheng, Zhang Shenfang. Application of Nursing Management in Preventing and Controlling Hospital Infection in Cancer Patients[J]. Chinese Journal of Nosocomiology, 2012, (10): 2136-2137.

[12] Tian Duoqin. Experience in the management of infection in operating room [J]. Endemic Disease Bulletin, 2008, 23 (03): 93-94.

[13] Heng Ruirui. The effect of nursing risk management on the control of nosocomial infections [J]. Jilin Medical Journal, 2015, (07): 1468-1469. 